\title{
Study on Classification of EEG Signals Based on Wavelet Transformation and BP Neural Network
}

\author{
Yu Zhulin ${ }^{1}$ Zhao Bing ${ }^{1}$ Liu Jie ${ }^{2}$ Yu Mingtao ${ }^{3}$ Xu Ling ${ }^{1}$
}

1. Department of Electrical and Mechanical Engineering, Yantai Engineering and Technology College, Yantai 264006, China;

\section{Department of Aerocraft Engineering, Naval Aeronautical and Astronautical University, Yantai} 264001

3. Medical Equipment Department, Yantaishan Hospital, Yantai 264001, China

Author brief introduction: Zhulin Yu (1981 -), male, Shandong Yantai people, master, lecturer, engaged in teaching and research in control theory and control technology .

Contact: 15064597308, yuzhulin1125@126.com

Keywords: Electroencephalography Signal; Wavelet Transformation; BP Neural Network

\begin{abstract}
The method of wavelet transformation and BP neural network for classification of epileptic intermission and epileptic attack signals was studied. The discrete binary wavelet transformation of EEG signal was carried out with db4 wavelet function. The third to fifth level signals by reconstruction and noise reduction were extracted as feature signals. The BP neural network with three levels was built for signal classification by the variation coefficient and fluctuation index. The accuracy recognition rate of EEG signals reaches $95 \%$ in simulation experiment. The results show that the method on classification of epileptic intermission and epileptic attack signals is effective and rapid.
\end{abstract}

\section{Introduction}

EEG signals was the voltage change of the cerebral cortex or the surface of the scalp which was caused by the brain electrophysiological activities[1],which was recorded through the electrodes. EEG signals containsed a large number of physiological and pathological information, it may provide the basis for the clinical diagnosis and treatment of brain disease through the analysis and processing of EEG signals.it is a non-stationary random signal under strong noise background $^{[2]}$, which contains the ingredients are very complicated. To diagnose the disease by using EEG signal,the effective information of singals must be extracted by using the appropriate signal processing methods for EEG classification $\left.{ }^{[3-4}\right]$. At present there are many methods for EEG signal processing, including wavelet analysis method which is very suitable for noisy nonstationary EEG signalinformation extraction ,because it can make use of the multi resolution characteristics of multi-scale decomposition of signals,extracting time domain features band limited signal,at the same time to meet the requirements of analysis in frequency domain and time domain. According to the epileptic intermission and epileptic attack signals,this paper uses the method of wavelet transform to remove noise interference and extract Characteristics of EEG signal which are classified by BP neural network method,so as to identify the EEG signal.

\section{The wavelet transform of EEG signals}

Wavelet transform can decompose the EEG signal into multiple scales, Scales of effective signal and interference signal with different singularity can removal characteristics of noise and keep the effective signal. The main frequency range of epileptic EEG signals is between 3hz and 25hz, The high frequency signal is higher than that of $3-25 \mathrm{hz}$ reflected in the low scale after decomposition,can directly filter. Interference components, whitch are overlapped with the signal frequency in other scales , are filtered by setting the appropriate wavelet threshold. This paper uses the discrete binary wavelet transformation for signal processing, The treatment process is divided 
into two steps:

(1)The wavelet transform on EEG signals can get the layers of wavelet coefficients, according to the characters of brain electric signal to choose the appropriate wavelet functions and decomposition layers.

(2) Select the appropriate threshold processing method to eliminate the noise in the low frequency wavelet coefficients of elements

After denoising the scale wavelet coefficients of signals as the electrical characteristics, calculation process by Mallat algorithm quickly, in the process of main parameters for wavelet basis function and threshold, according to the following principles to select and Settings

(1)The wavelet basis function

Used in signal processing of the wavelet basis function is usually consistent with the signal characteristics 。 Often used in signal processing of wavele function has $\mathrm{dbN}$ wavelet, symN wavelet and coifN wavelet, Use them for correlation calculation with epilepsy wave , the results show that daubechies4(db4) wavelet maximum value associated with epilepsy wave, So, choose the db4 wavelet as eeg signals processing of wavelet base

(2)The threshold value

Threshold selection methods are divided into hard threshold and soft threshold method. After soft threshold denoising process the signal is more smooth. There are four main threshold selection rules of Soft threshold method, sqtwolog,Stein, heursure, minimaxi $)^{[5]}$, stein and minimaxi less risk, and are suitable for separation of weak signal from the noise, when the high frequency components of signals have overlap with noise. Beause the high frequency components of brain electrical signal exist overlap with noise, so choose threshold estimation with Stein can to avoid filtering effective signal.

\section{The eigenvalue extraction of EEG}

The amplitude of EEG signals of epileptic attack is usually higher than that of epileptic intermission .In addition, there exists obvious differences on the frequency of the signal fluctuation between epileptic attack and epileptic intermission. Therefore, use the variation coefficient and fluctuation index which can reflect the characteristics of the above signals as the characteristic value of classification of EEG signals, according to the following method to calculate respectively

a formula ${ }^{[6]}$ to calculate of variation coefficient

$$
c=\frac{\sigma^{2}}{\mu^{2}}
$$

among them, $\sigma$ for Standard deviation of the signal, $\mu$ for the mean signal a formula to calculate of fluctuation index

$$
F=\frac{1}{M} \sum_{n=1}^{M}|x(n+1)-x(n)|
$$

among them, $\mathrm{M}$ for signal data points, $\mathrm{x}(\mathrm{n})$ for the $\mathrm{N}$ points signal value

Electrical characteristic signal which is extracted by wavelet transform according to the formula (1), (2) were used to calculate the signal variation coefficient and fluctuation index which are as to characteristic parameters of signal classification

\section{The classification of the EEG signals}

BP artificial neural network has good nonlinear mapping approximation ability and the ability to predict, a lot of information can be stored in neurons, by the known training samples, to predict unknown samples $^{[7]}$. For brain electrical signal containing complex information,BP neural network can establish the relationship between characteristic parameters and classification results by known samples, which can realize prediction for the classification of the EEG signals.

The network which consists of input layer,hidden layer and output layer of three parts is a 
feedforward multilayer neural network,which make the network convergence by repeated alternation of network training of mode propagation and error back propagation. This paper, by using the MATLAB neural network toolbox to build a three layers BP neural network for the classification of the EEG signals, Selection and Settings of network main parameters are as follows:

(1)The number of nodes in each layer

Variation coefficient and fluctuation index vector of Characteristics of eeg signals are as the input parameters of the network ,so the input layer contains two neurons. Output variables for eeg classification results, ' 0 ' and ' 1 ' respectively represent the epileptic attack and epileptic intermission signals, so the output layer contains a neuron According to the experience to determine the number of hidden layer nodes, shoulds not be too much or too little.More the number of hidden layer nodes can store more information, but may extend the training time, conversely, too little number of hidden layer nodes cannot correctly reflect the relationship between input and output variables, and in the process of the training is easy to fall into local minimum

(2)The transfer function

Transfer function of hidden layer use the nonlinear function which apply more with Sigmoid function and Sigmoid function Due to the sample output is greater than or equal to zero, so the selection of commonly used Sigmoid function as the transfer function of hidden layer .To maintain the range of output, the output layer adopts linear Purelin function

(3) Training function

Due to establish the number of layers of BP neural network and the node number is less, so choose suitable for small networks, fast convergence and small error, Levenberg - Marquardt function for the training function

\section{The simulation results}

This article uses the eeg data from database of epilepsy of Bonn, Germany, epilepsy research ,the epileptic intermission and epileptic attack signals are chosen as 200 segments, the sampling frequency is $173.61 \mathrm{~Hz}$, band-pass filter was $0.5 \sim 85 \mathrm{~Hz}$, the sampling time for $1 \mathrm{~s}$. By adopting $\mathrm{db} 3$ wavelet on the original eeg signals in a 5 layer of wavelet transform, The signal frequency range the $3 \sim 5$ wavelet coefficients after reconstruction for $2.5 \sim 22 \mathrm{~Hz}$, the main frequency range is located within the epileptic waves. On the basis of the Stein soft threshold method to estimate reconstructed signal threshold of each layer and signal denoising, to respectively get the EEG signals of the frequency range of $11 \sim 22 \mathrm{~Hz}, 5 \sim 11 \mathrm{~Hz}$ and $2.5 \sim 5 \mathrm{~Hz}$. The original EEG signals of this section and the filter noise signal of the $3 \sim 5$ layer after wavelet transform are as shown in figure 1 , 2

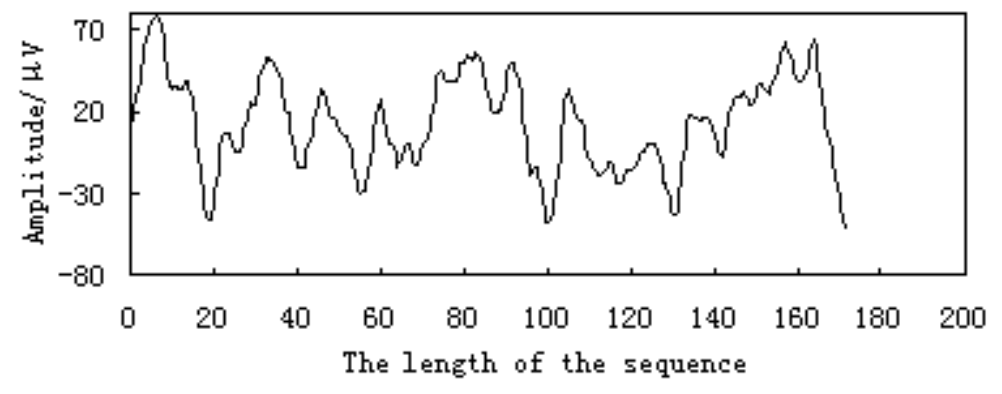

Figure 1 original eeg signals 


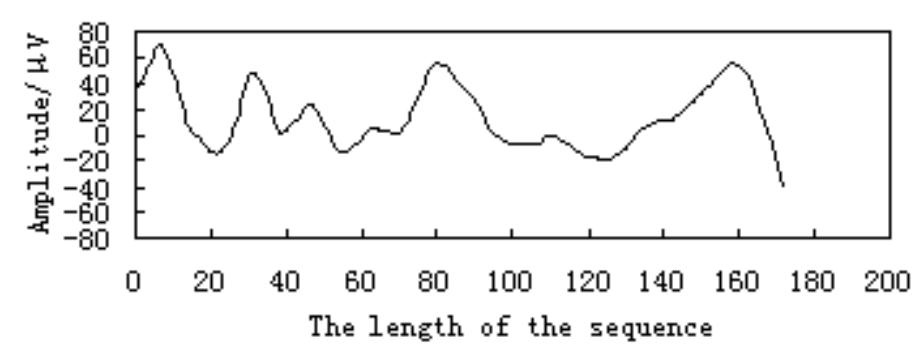

(a) Layer 3

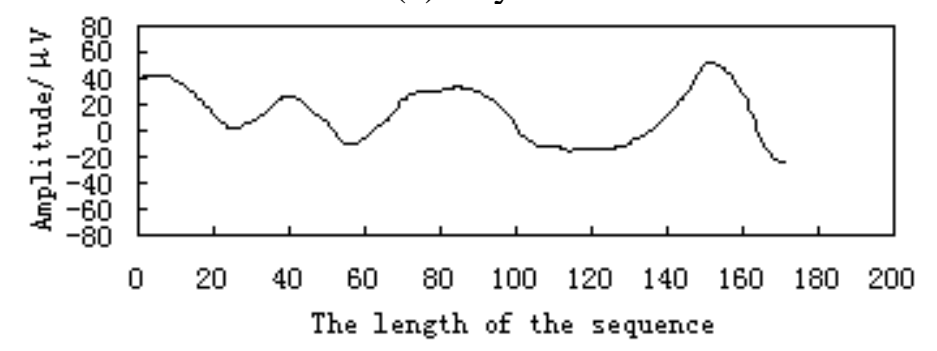

(b) Layer 4

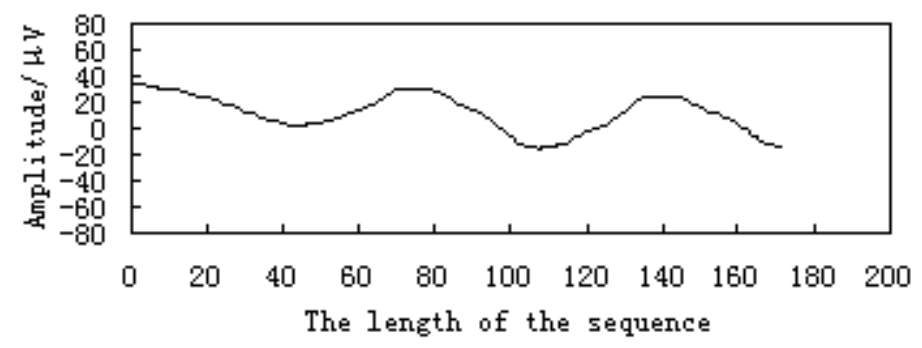

(c) Layer 5

Figure 2 the characteristics of EEG signals by wavelet transform

As can be seen from Figure 2, the original EEG signal based on wavelet transform andreconstruction after each layer is divided into different frequency, the frequency decreases with the increase of layers, so is beneficial to extract effective signal of specific frequency. The filtered signal is smooth in each layer, and more fully reflect the characteristics of the signal, Variation coefficient and fluctuation index of each layer signal are calculated by formula (1), (2), the results are as shown in Table 1

Table 1 variation coefficient and fluctuation index of each layer signals

\begin{tabular}{ccc}
\hline layer & variation coefficient & fluctuation index \\
\hline 3 & 23.18 & 3.5775 \\
4 & 20.03 & 1.9739 \\
5 & 14.05 & 1.0843 \\
\hline
\end{tabular}

The 200 section of epileptic intermission and 200 section of ictal EEG signals are respectively preprocessed according to the same method。Signal variation coefficient and fluctuation index of 3 5 layer are obtained by each section of signal after wavelet transform, which are composed of two column vectors as the input parameters of BP neural network。Among them, 150 coefficient of variation and fluctuation index vector are as training set of BP neural network as, the rest for the validation set 。 According to the method to establish the BP neural network, using Matlab toolbox to build a three layer BP neural network, the hidden layer transfer function is 'logsig', the output layer transfer function is' purelin ', the training function is ' trainelm '. Setting the network training error goal is 0.01 , the maximum training step is 5000 . The input layer neurons number is 2 , the output layer neurons number is 1 , the optimized number of neurons in hidden layer is 5 through the network training speed and classification accuracy. Through the training set for the 34 step of the network training error is 0.004443 , meet the target error. The simulation of the validation set using 
the network, of which 50 section of epileptic intermission signal and 50 section of ictal signal's accurately identify are respectively the number of 48 cases and 47 cases, namely, the recognition rate are respectively $96 \%$ and $94 \%$. The overall recognition rate of $95 \%$.

\section{Conclusion}

Method combining wavelet transform and BP neural network is used to identify the epileptic intermission and ictal of EEG signals, adaptive of wavelet transform is used to extract the effective signal frequency information. thus, accurate classification of signals is realized by using BP neural network. Simulation results show that the method can accurately distinguish epileptic intermission and ictal EEG signals, And signal classification by using the effective information can reduce the data length of input parameters of neural network, reduce the complexity of network structure, improve the discriminative rate. This method has a certain practical value in the EEG signal automatic detection and prediction of epileptic attack

\section{Reference}

[1] Zhang Renlong,Ma Wenli,Yao Wenjuan, Zheng Wenling,Liang Bin.Analysis and processing of EEG signal based on wavelet packet transforman[J].ELECTRONIC MEASUREMENT TECHNOLOGY,2007,30(3):22-24

[2] SHEN M,SUN L,CHAN F H Y. Method for Extracting Time-varying Rhythms of Electroencephalogy Via Wavelet Packet Analysis[J].IEE Proceedings, Science, Measurement and Technology,2001,148(1):23-27.

[3] Ji Zhong,Qin Shu ren.The Application of Time-Frequency Testing Methods in EEG Signals Analysis[J].Jouranl of Chongqing University,2003,26(11):1-5.

[4] Dong Meng meng,ZhongYi,Xu Jie.Analysis of EEG signals based on wavelet threshold de-noising[J].Electronic Design,Engineering,2012,20(24):59-61

[5] Jiang Ying chun. The theory of wavelet analysis [M].Tian jin,Press of Tianjin University,2012.

[6] Zheng Fang,Xu Ming xing. Principles of signal processing [M].Beijing:press of Tsinghua University ,2007.

[7] Han Li qun. Course of the artificial neural network [M].Beijing: Beijing University of Posts and Telecommunications press, 2006. 